\title{
Structure and Immune Recognition of the Porcine Epidemic Diarrhea Virus Spike Protein
}

Robert Kirchdoerfer

University of Wisconsin-Madison, Madison, Wisconsin, United States

Porcine epidemic diarrhea virus (PEDV) is an alphacoronavirus responsible for an enteric infection in pigs. This pathogen is highly transmissible and especially lethal to neonatal piglets with mortalities approaching $100 \%$. PEDV has a global prevalence and represents a significant economic burden in losses to the pork industry. Coronaviruses enter cells using their spike proteins to bind to host receptors and mediate the fusion of virus and host membranes and release viral genomic material into infected cells. As the major protein on the surface of virions, the PEDV spike protein is also a key target for the host antibody response and a good candidate for a protein-based vaccine immunogen. We used single-particle electron microscopy to evaluate the PEDV spike structure, as well as pig polyclonal antibody responses to viral infection. The cryo-electron microscopy structure of the PEDV spike determined to $3.5 \AA$ reveals a domain configuration similar to that of HuCoV-NL63. In the structure determined here, the PEDV S1 domain 0 lies adjacent to the $\mathrm{S} 2$ fusion machinery in contrast to a previously determined PEDV spike structure. We further determined that this alternate conformation is not a result of differing recombinant expression systems or glycosylation patterns. Several PEDV spike domain interfaces are mediated by non-protein components including a glycan at Asn264 and two bound palmitoleic acid molecules. To examine the spike polyclonal antibody response to PEDV infection, we isolated spikes bound to polyclonal Fab and performed negative-stain electron microscopy. Extensive sorting of Fab-bound particles shows a predominance of epitopes in the spike $\mathrm{S} 1$ region. This includes an epitope to $\mathrm{S} 1$ receptor binding domains as well as a known neutralizing epitope on $\mathrm{S} 1$ subdomains both of which are convergent responses across three independent porcine sera samples. This structural and immune characterization provides new insights into coronavirus spike stability determinants and explores the immune landscape of viral spike proteins.

\section{References}

Bianchi, M., Turner, H.L., Nogal, B., Cottrell, C.A., Oyen, D., Pauthner, M., Bastidas, R., Nedellec, R., McCoy, L.E., Wilson, I.A., et al. (2018). Electron-Microscopy-Based Epitope Mapping Defines Specificities of Polyclonal Antibodies Elicited during HIV-1 BG505 Envelope Trimer Immunization. Immunity 49, 288-300.e288.

Jung, K., and Saif, L.J. (2015). Porcine epidemic diarrhea virus infection: Etiology, epidemiology, pathogenesis and immunoprophylaxis. Vet J 204, 134-143.

Walls, A.C., Tortorici, M.A., Frenz, B., Snijder, J., Li, W., Rey, F.A., DiMaio, F., Bosch, B.J., and Veesler, D. (2016). Glycan shield and epitope masking of a coronavirus spike protein observed by cryo-electron microscopy. Nat Struct Mol Biol 23, 899-905.

Wrapp, D., and McLellan, J.S. (2019). The 3.1 A cryo-EM structure of the porcine epidemic diarrhea virus spike protein in the prefusion conformation. J Virol. 